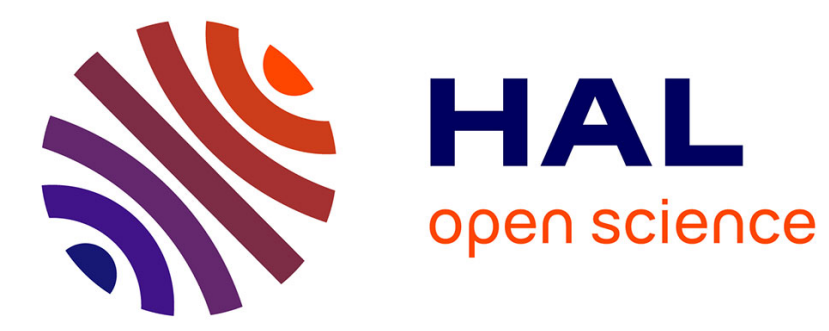

\title{
Statistical Modelling of Temporal Dependence in Financial Data via a Copula Function
}

Filippo Domma, Sabrina Giordano, Pier Francesco Perri

\section{To cite this version:}

Filippo Domma, Sabrina Giordano, Pier Francesco Perri. Statistical Modelling of Temporal Dependence in Financial Data via a Copula Function. Communications in Statistics - Simulation and Computation, 2009, 38 (04), pp.703-728. 10.1080/03610910802645321 . hal-00514343

\section{HAL Id: hal-00514343 \\ https://hal.science/hal-00514343}

Submitted on 2 Sep 2010

HAL is a multi-disciplinary open access archive for the deposit and dissemination of scientific research documents, whether they are published or not. The documents may come from teaching and research institutions in France or abroad, or from public or private research centers.
L'archive ouverte pluridisciplinaire HAL, est destinée au dépôt et à la diffusion de documents scientifiques de niveau recherche, publiés ou non, émanant des établissements d'enseignement et de recherche français ou étrangers, des laboratoires publics ou privés. 


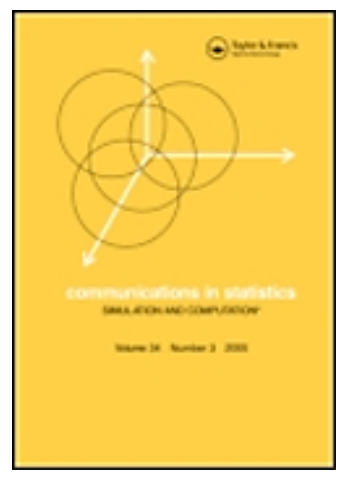

\section{Statistical Modelling of Temporal Dependence in Financial Data via a Copula Function}

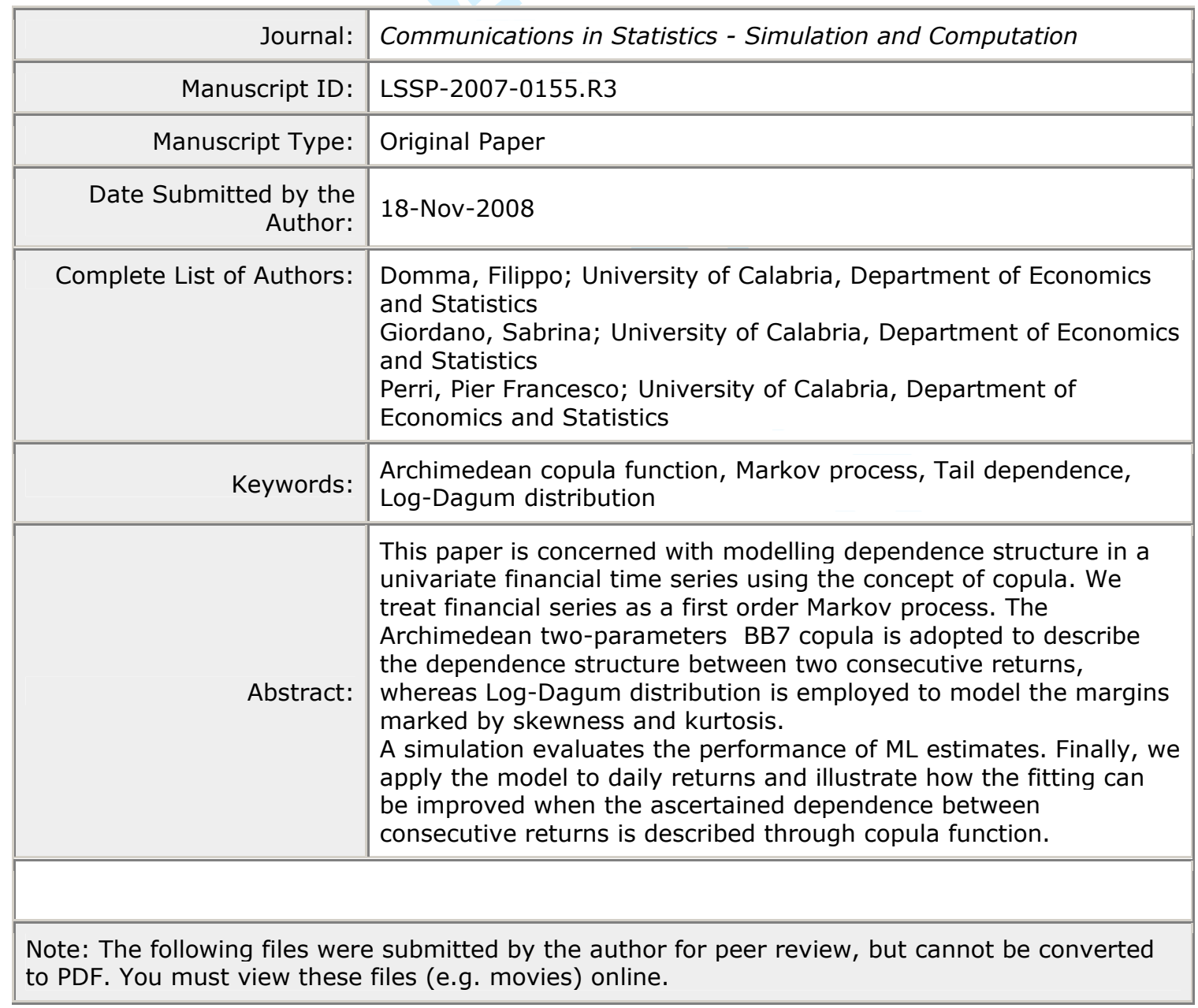


1

2

3

4

5

6

7

8

9

10

11

12

13

14

15

16

17

18

19

20

21

22

23

24

25

26

27

28

29

30

31

32

33

34

35

36

37

38

39

40

41

42

43

44

45

46

47

48

49

50

51

52

53

54

55

56

57

58

59

60

Domma-Giordano-Perri.rar

\section{ScholarONE \\ Manuscript Central}

URL: http://mc.manuscriptcentral.com/Issp E-mail: comstat@univmail.cis.mcmaster.ca 


\title{
Statistical Modelling of Temporal Dependence in Financial Data via a Copula Function
}

\author{
Modelling temporal dependence via copula \\ Filippo Domma* Sabrina Giordano \\ Pier Francesco Perri \\ Department of Economics and Statistics \\ University of Calabria \\ Via Pietro Bucci, Cubo 0C \\ 87036 Arcavacata di Rende (CS) - Italy -
}

* Corresponding Author: Filippo Domma, f.domma@unical.it 
Key words: Log-Dagum distribution, Archimedean copula function, Markov process, Tail dependence, Returns.

\section{Introduction}

The stylized facts (Cont, 2001) which characterize financial markets have been the focus of several studies, many of which have emphasized the fact that the empirical distribution of financial returns is far from the Normal model since it tends to be asymmetric and heavy-tailed. As alternatives to the classical Gaussian model, a few parametric distributions of financial assets, allowing for skewness and fat tails, have been proposed in the literature. The choice among these is usually a matter of analytical and numerical tractability.

It is generally unrealistic to assume independence between consecutive observations in financial time series. For example, financial data may show a temporal dependence structure of its volatility which is often referred to as volatility clustering: large (small) absolute returns tend to follow large (small) absolute returns. Indeed, there may exist different forms of nonlinear dependence which need to be properly modelled. Since the seminal paper by Engle (1982), a wide variety of ARCH-type models has been introduced to take into account the volatility clustering. However, the traditional assumption of independent and identically distributed (i.i.d.) innovations carried out in the ARCHtype model seems to be inappropriate (Bingham and Schmidt, 2005). 
Recently, the copula function has been employed to overcome the problem of modelling simultaneous dependence between two or more time series. Copulas have already been widely used in finance and economics since one can describe any multivariate distribution by modelling its margins and its copula separately (see, e.g., Frees and Valdez, 1998; Klugman and Parsa, 1999; Embrechts et al., 2003; Cherubini et al., 2004; Hürlimann, 2004; Mendes and Souza, 2004).

Besides the simultaneous dependence between several time series, it is also important to model (nonlinear) temporal dependence in a univariate time series. One possibility is to treat the time series as a Markov process. To model a stationary Markov process, Joe (1997) described a parametric approach for both copulas and marginal distributions. This author considered a wide class of copulas and applied a one-parameter copula to daily environmental data.

Bouyé et al. (2002) investigated the dynamic dependence in non-Gaussian time series using different types of Archimedean copula functions for nonlinear autoregressive dependence. Chen and Fan (2006) proposed to capture nonlinear temporal dependence in univariate time series through a class of stationary Markov models characterized by nonparametric marginal distributions and parametric copula functions. Savu and $\mathrm{Ng}$ (2005) applied Chen and Fan semiparametric copula approach to modelling the duration of ultra-high-frequency data as an alternative to the autoregressive conditional duration model.

In order to overcome limits connected with independence and normality, in this paper, we propose to model - in a univariate time series - the dependence between two consecutive financial returns using a copula approach with skewed and heavy-tailed margins. We mainly follow Joe (1997) in adopting a parametric approach for both the marginal distributions and the copula function. A two-parameter Archimedean copula family, called BB7, is chosen to describe the temporal dependence in a Markov process. Then, the marginal returns are modelled through the log-Dagum distribution (Domma and Perri, 2009) that allows for skewness and heavy tails.

The remaining part of the paper is structured as follows. Section 2 is devoted to the copula approach: the BB7 copula function and its main features are introduced emphasizing various measures of dependence and the salient characteristics of the log-Dagum model. In Section 3 we deal with the maximum likelihood estimates of the model. A simulation study, assessing the stability of the estimation procedure, is carried out in Section 4, while in Section 5 we report the results of an empirical study performed on four real time series, point out some stylized facts and fit the BB7 copula approach to data. Finally, Section 6 summaries the work and offers some suggestions for further research. 


\section{The Copula Approach}

The aim of this section is to construct a model for financial returns which allows for temporal dependence, skewness and tail heaviness. In describing one-dimension time series we need to model the temporal dependence and the univariate margins. In so doing, it is useful to introduce notation and some preliminary results. More specifically, in the first part of the section we illustrate how the general properties of the copula function make it a suitable tool for modelling dependence between two or more random variables. We focus on the two-parameter Archimedean BB7 copula and provide original results on ordering dependence properties as well as on the derivation of dependence measures such as Kendall's tau and the medial correlation coefficient (Blomqvist, 1950). In the second part of the section, we apply the copula approach to the distribution of financial returns. In particular, following Joe (1997), we attempt to describe the behavior of a univariate time series through a first order Markov process whose components are log-Dagum distributed.

\subsection{Basic concepts}

The copula is a multivariate distribution with Uniform $(0,1)$ margins. Key references on this topic are Nelsen (1999), which is a excellent primer, and Joe (1997) which provides a comprehensive review from a mathematical perspective. For the aim of this paper, we will consider a two-dimensional copula and, without loss of generality, introduce notation for bivariate distributions.

It is known that the dependence between the random variables $X$ and $Y$ is fully described by the joint distribution function

$$
F_{X Y}(x, y)=P(X \leq x, Y \leq y)
$$

The idea of separating $F_{X Y}(x, y)$ in two parts, one which refers to the dependence structure and the other which describes the marginal behavior only, leads to the concept of copula. A bivariate copula, defined on the unit square $I^{2}=[0,1] \times[0,1]$, is a bivariate distribution function with univariate Uniform margins on $I$. The connection between the joint distribution function of two random variables and their margins through a copula is established by Sklar's theorem. Let $X$ and $Y$ be continuous random variables with distribution function $F_{X}(x)$ and $G_{Y}(y)$ and joint distribution function $F_{X Y}(x, y)$, respectively. Then, there exists a copula $C$ such that for all $(x, y)$ in $\mathbb{R}^{2}$

$$
F_{X Y}(x, y)=C\left(F_{X}(x), G_{Y}(y)\right)
$$

If $F_{X}(x)$ and $G_{Y}(y)$ are continuous, $C$ is unique.

The converse of Sklar's theorem is particularly useful for modelling bivariate distribution because it implies that any group of univariate distributions can be joined together with different copulas to 
define a valid multivariate distribution. This means that (1) can be rewritten to provide a method for obtaining a copula from the joint distribution function $F_{X Y}(x, y)$

$$
C(u, v)=F_{X Y}\left(F_{X}^{-1}(u), G_{Y}^{-1}(v)\right)
$$

where $F_{X}^{-1}(u)$ and $G_{Y}^{-1}(v)$ are the inverse functions of the two margins and $(u, v) \in I^{2}$.

In addition to the copula function, conditional and density copula are also defined. The first derivative of the copula function, for instance with respect to $u$, yields the conditional copula of $V$ given $U=u$

$$
C_{2 \mid 1}(v \mid u)=\frac{\partial C(u, v)}{\partial u} .
$$

The expression of $C_{1 \mid 2}(u \mid v)$ is defined accordingly. Furthermore, the density copula is given by

$$
c(u, v)=\frac{\partial^{2} C(u, v)}{\partial u \partial v}
$$

Copulas offer a natural way to study and measure dependence between random variables. There exist different dependence measures useful in the applications. For instance, Kendall's tau

$$
\tau_{X Y}=P\left[\left(X_{1}-X_{2}\right)\left(Y_{1}-Y_{2}\right)>0\right]-P\left[\left(X_{1}-X_{2}\right)\left(Y_{1}-Y_{2}\right)<0\right]
$$

defined as probability of discordance of two pairs of random vectors, say $\left(X_{1}, Y_{1}\right)$ and $\left(X_{2}, Y_{2}\right)$, and described by the same joint bivariate distribution $F_{X Y}(x, y)$, can be written as

$$
\tau_{X Y}=4 \int_{I^{2}} C(u, v) \frac{\partial^{2} C(u, v)}{\partial u \partial v} d u d v-1
$$

Moreover, the medial correlation coefficient for a pair $(X, Y)$ of continuous random variables

$$
\mathcal{M}_{X Y}=P\left[\left(X-\mathcal{M}_{X}\right)\left(Y-\mathcal{M}_{Y}\right)>0\right]-P\left[\left(X-\mathcal{M}_{X}\right)\left(Y-\mathcal{M}_{Y}\right)<0\right]
$$

where $\mathcal{M}_{X}$ and $\mathcal{M}_{Y}$ denote the medians of $X$ and $Y$, can be easily expressed as

$$
\mathcal{M}_{X Y}=4 F_{X Y}\left(\mathcal{M}_{X}, \mathcal{M}_{Y}\right)-1=4 C\left(\frac{1}{2}, \frac{1}{2}\right)-1
$$

In many fields, such as finance, insurance and economics, one important aspect is the dependence in the tails of a multivariate distribution. Tail dependence describes the behavior of the variables when extreme events occur. Intuitively, upper (lower) quadrant tail dependence is defined as limiting probability that one margin exceeds (does not exceed) a certain threshold given that the other margin has already exceeded (has not already exceeded) a threshold. 
The most common measures of tail dependence are the tail dependence coefficients. It is wellknown that $(X, Y)$ is upper tail dependent if the following limit exists and is positive

$$
\lambda_{U}=\lim _{p \rightarrow 1^{-}} P\left[X>F_{X}^{-1}(p) \mid Y>F_{Y}^{-1}(p)\right] .
$$

Consequently, $(X, Y)$ is upper tail independent if $\lambda_{U}=0$. The quantity $\lambda_{U}$ is the upper tail dependence coefficient.

Similarly, the lower tail dependence coefficient is defined as

$$
\lambda_{L}=\lim _{p \rightarrow 0^{+}} P\left[X<F_{X}^{-1}(p) \mid Y<F_{Y}^{-1}(p)\right]
$$

and $(X, Y)$ is said to be lower tail dependent if $\lambda_{L} \in(0,1]$. It is worth pointing out that, in general, copula families depend on one or more parameters, called association parameters. Most of the traditional dependence measures are functions of these parameters. However, in many situations we need models that allow for a different degree of lower and upper tail dependence. For instance, in finance or insurance, more emphasis is given to dependence between extreme losses than between extreme gains. This kind of asymmetry in the tail dependence can be modelled, for instance, through Archimedean copulas.

\subsection{The Archimedean BB7 copula}

The Archimedean family of copulas has a wide range of applications in different fields. Its common use stems from the fact that they are easy to construct and possess many nice properties (see Nelsen, 1999; Joe, 1997). Each family belonging to this class can be expressed by

$$
C(u, v)=\varphi^{-1}(\varphi(u)+\varphi(v))
$$

where $\varphi(t):[0,1] \rightarrow[0, \infty)$ is the generator function that is continuous, strictly decreasing, convex and $\varphi(1)=0$.

Although several common one-parameter Archimedean copulas have been extensively applied, they are not suitable to describe tail dependence because the lower and upper tail coefficients are, in general, expressed as functions of the unique association parameter. The possibility of distinguishing the dependence in the upper quadrant tail from that in the lower tail was the key criterion which led to the choice of the copula. In particular, the use of Archimedean copulas, in this paper, is restricted to the family named BB7 in Joe's classification (Joe, 1997)

$$
C(u, v ; \theta, \delta)=1-\left\{1-\left[\left(1-\bar{u}^{\theta}\right)^{-\delta}+\left(1-\bar{v}^{\theta}\right)^{-\delta}-1\right]^{-\frac{1}{\delta}}\right\}^{\frac{1}{\theta}}, \quad \theta \geq 1 ; \delta>0
$$


where $\bar{u}=1-u, \bar{v}=1-v$ and generator function $\varphi(t)=\left[1-(1-t)^{\theta}\right]^{-\delta}-1$. The lower and upper tail dependence for the BB7 copula are $\lambda_{L}=2^{-1 / \delta}$ and $\lambda_{U}=2-2^{1 / \theta}$, respectively. It is worth emphasizing that parameter $\theta$ allows us to capture the upper tail dependence only, whereas $\delta$ is related to the lower tail dependence. For this characteristic, the BB7 copula plays an important role among all the two-parameter Archimedean copulas.

For the methodological developments that we will focus on later, we now report the conditional and density BB7 copula

$$
C_{2 \mid 1}(v \mid u ; \theta, \delta)=\left(1-\omega^{-\frac{1}{\delta}}\right)^{\frac{1}{\theta}-1} \omega^{-\frac{1}{\delta}-1}\left(1-\bar{u}^{\theta}\right)^{-\delta-1} \bar{u}^{\theta-1}
$$

and

$$
\begin{aligned}
c(u, v ; \theta, \delta)= & \left(1-\bar{u}^{\theta}\right)^{-\delta-1} \bar{u}^{\theta-1}\left(1-\bar{v}^{\theta}\right)^{-\delta-1} \bar{v}^{\theta-1}\left(1-\omega^{-\frac{1}{\delta}}\right)^{\frac{1}{\theta}-2} \\
& \times \omega^{-\frac{2}{\delta}-2}\left[(\theta-1)+\theta(\delta+1)\left(\omega^{\frac{1}{\delta}}-1\right)\right] .
\end{aligned}
$$

where $\omega=\left(1-\bar{u}^{\theta}\right)^{-\delta}+\left(1-\bar{v}^{\theta}\right)^{-\delta}-1$.

These aspects are now integrated with our findings on dependence properties and measures.

Joe (1997) asserts that the concordance of the BB7 copula increases as $\theta$ rises when $\delta \leq 1$ and conjectures that the concordance also increases in $\theta$ when $\delta>1$. Here we provide some results regarding Stochastic Monotonicity. In order to prove that the BB7 family satisfies the Stochastically Increasing Ordering, we start from the result of Capéraà and Genest (1993): if $\varphi^{-1}$ is differentiable, then $Y$ is stochastically increasing in $X$ or $X$ is stochastically increasing in $Y$ if and only if $g(t)=\ln \left(-\frac{\partial \varphi^{-1}(t)}{\partial t}\right)$ is convex on $(0, \infty)$. Indeed, with the BB7 copula, it is a simple matter to verify that

$$
\frac{\partial^{2} g(t)}{\partial t^{2}}=\frac{(\delta+1)}{\delta(1+t)^{2}}+\frac{(\theta-1)\left\{\delta(1+t)^{-2}\left[(1+t)^{\frac{1}{\delta}}-1\right]+(1+t)^{\frac{1}{\delta}-2}\right\}}{\delta^{2} \theta\left[(1+t)^{\frac{1}{\delta}}-1\right]^{2}}>0
$$

for any $t>0, \delta>0$ and $\theta>1$. We remind the reader that the Stochastic Monotonicity implies the Tail Monotonicity properties and this satisfies the Positively Quadrant Dependence properties. Consequently, the correlation coefficient $(\rho)$, Kendall's tau $(\tau)$, Spearman's rho $\left(\rho_{S}\right)$ and the medial correlation coefficient $(\mathcal{M})$ all assume non-negative values (Lehmann, 1966).

Now we revisit two measures of dependence in terms of the two parameters of the BB7 copula.

Proposition. Kendall's tau index for copula BB7 is given by

$$
\tau_{X Y}(\theta, \delta)=1-\frac{4}{\delta \theta^{2}}\left[B\left(2, \frac{2}{\theta}-1\right)-B\left(\delta+2, \frac{2}{\theta}-1\right)\right]
$$


for $\delta>0$ and $\theta<2$, where $B(p, q)$ is the Beta function.

Proof. For Achimedean copulas, Kendall's tau can be written (Joe, 1997) as

$$
\tau_{X Y}=1-4 \int_{0}^{\infty} u\left[\frac{\partial \varphi^{-1}(u)}{\partial u}\right]^{2} d u .
$$

For the BB7 copula

$$
\frac{\partial \varphi^{-1}(u)}{\partial u}=-\frac{1}{\theta \delta}(1+u)^{-\frac{1}{\delta}-1}\left[1-(1+u)^{-\frac{1}{\delta}}\right]^{\frac{1}{\theta}-1}
$$

which, replaced in (3), gives

$$
\tau_{X Y}(\theta, \delta)=1-\frac{4}{\delta^{2} \theta^{2}} \int_{0}^{\infty} u(1+u)^{-\frac{2}{\delta}-2}\left[1-(1+u)^{-\frac{1}{\delta}}\right]^{\frac{2}{\theta}-2} d u
$$

Setting first $y=1+u$ and then $x=y^{-\frac{1}{\delta}}$, after some algebra, we get

$$
\begin{aligned}
\tau_{X Y}(\theta, \delta) & =1-\frac{4}{\delta \theta^{2}}\left[\int_{0}^{1} x(1-x)^{\frac{2}{\theta}-2} d x-\int_{0}^{1} x^{\delta+1}(1-x)^{\frac{2}{\theta}-2} d x\right] \\
& =1-\frac{4}{\delta \theta^{2}}\left[B\left(2, \frac{2}{\theta}-1\right)-B\left(\delta+2, \frac{2}{\theta}-1\right)\right] .
\end{aligned}
$$

As regards the medial correlation, after simple algebra, it is possible to show that

$$
\mathcal{M}_{X Y}(\theta, \delta)=3-4\left\{1-\left[2\left(1-2^{-\theta}\right)^{-\delta}-1\right]^{-\frac{1}{\delta}}\right\}^{\frac{1}{\theta}} .
$$

In Figure 1 the behavior of Kendall's tau and the medial correlation coefficient is illustrated. Both the indices show that the amount of dependence increases as either $\theta$ and $\delta$ increase.

\subsection{Copula and Markov process}

The concepts earlier introduced for a generic variable $(X, Y)$ are now adapted to the case of $\left(Y_{t-1}, Y_{t}\right)$ which are two consecutive random variables of a stationary Markov process.

Let us assume $\left\{Y_{t}: t=1,2, \ldots, T\right\}$ to be a first order stationary Markov process with continuous state space. Then, its properties are completely determined by the joint distribution functions of $Y_{t-1}$ and $Y_{t}$, say $F\left(y_{t-1}, y_{t}\right)$. From Sklar's theorem, we can express $F\left(y_{t-1}, y_{t}\right)$ in terms of the marginal distribution function of $Y_{t}$ and the copula function of $Y_{t-1}$ and $Y_{t}$. In this way, the copula approach is suggested as a tool for modelling a stationary Markov process: instead of specifying the joint distribution function of $Y_{t-1}$ and $Y_{t}$ directly, one can specify the marginal distribution function of $Y_{t}$ and the copula function of $Y_{t-1}$ and $Y_{t}$. 

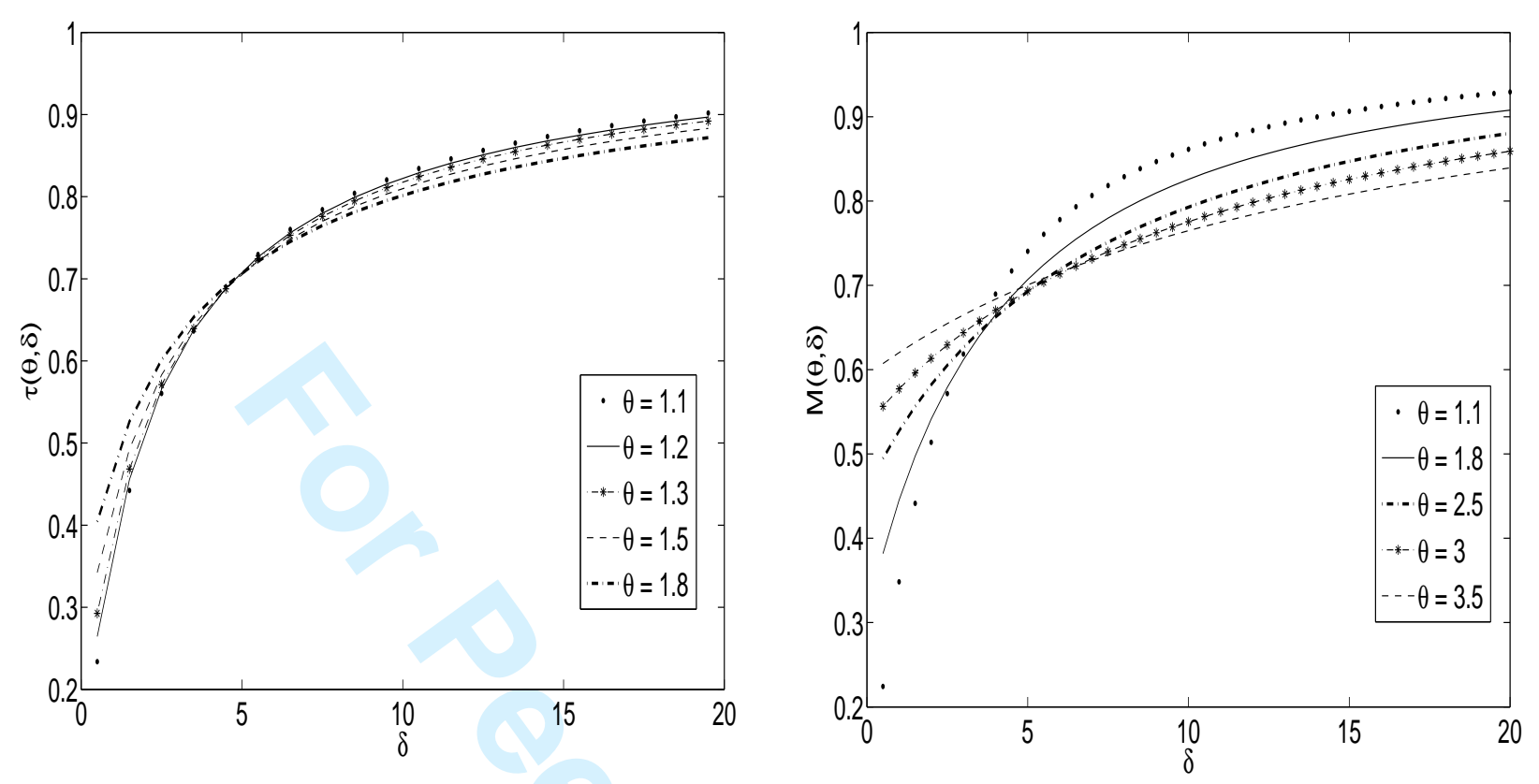

Figure 1: Kendall's tau and medial correlation coefficient.

From now on, we reserve the notation $Y_{t}$ to denote the financial return between $t$ and $t-1$

$$
Y_{t}=\ln P_{t}-\ln P_{t-1}
$$

where $P_{t}$ is the value of a financial asset (a stock, an exchange rate or a market index) at time $t$. The Markovian property seems to be an appropriate assumption for describing the behavior of the returns since it can be observed empirically that current returns are usually affected by their recent past. We restrict our attention to the first order process by assuming that only the immediate past is relevant for the current returns.

We consider that the return $Y_{t}$ and the lagged value $Y_{t-1}$ are random variables with identical continuous marginal distribution functions $F\left(y_{t} ; \boldsymbol{\alpha}\right)$ and $F\left(y_{t-1} ; \boldsymbol{\alpha}\right)$, for the hypothesis of stationarity, and joint distribution function given by

$$
F\left(y_{t-1}, y_{t} ; \boldsymbol{\alpha}, \boldsymbol{\xi}\right)=C\left(F\left(y_{t-1} ; \boldsymbol{\alpha}\right), F\left(y_{t} ; \boldsymbol{\alpha}\right) ; \boldsymbol{\xi}\right)
$$

where $\boldsymbol{\alpha}$ and $\boldsymbol{\xi}$ denote the vectors of marginal and association parameters, respectively. Our aim is to model the conditional behavior of $Y_{t}$ given its immediate past $Y_{t-1}$. In this sense, the conditional copula can be used to define the transition distribution function and the transition density function of $\left\{Y_{t}\right\}($ Joe, 1997)

$$
H\left(y_{t} \mid y_{t-1} ; \boldsymbol{\alpha}, \boldsymbol{\xi}\right)=P\left[Y_{t} \leq y_{t} \mid Y_{t-1}=y_{t-1}\right]=C_{2 \mid 1}\left(F\left(y_{t} ; \boldsymbol{\alpha}\right) \mid F\left(y_{t-1} ; \boldsymbol{\alpha}\right) ; \boldsymbol{\xi}\right)
$$




$$
h\left(y_{t} \mid y_{t-1} ; \boldsymbol{\alpha}, \boldsymbol{\xi}\right)=c\left(F\left(y_{t-1} ; \boldsymbol{\alpha}\right), F\left(y_{t} ; \boldsymbol{\alpha}\right) ; \boldsymbol{\xi}\right) f\left(y_{t} ; \boldsymbol{\alpha}\right) .
$$

In the sequel we will refer to this approach as the conditional approach, whereas the term marginal approach will be used for modelling $Y_{t}$ regardless of temporal dependence (that is under i.i.d. hypothesis). For the purpose of modelling the dependence of returns, we assume that $C(\cdot, \cdot ; \cdot)$ is the Archimedean BB7 copula function described in $\S 2.2$. Moreover, since returns tend to be asymmetric and heavy-tailed, we propose to model their marginal behavior through the log-Dagum distribution whose basic characteristics are summarized in the next section.

\subsection{Characteristics of the marginal distributions}

The log-Dagum random variable is obtained by a logarithmic transformation of Dagum random variable (Dagum, 1977, 1980). This distribution, recently investigated in Domma and Perri (2009), seems to be a flexible parametric family for modelling skewed and leptokurtic distributions, since it takes values on $\mathbb{R}$ and the shape is always leptokurtic. Moreover, it can be both symmetric and asymmetric (positive or negative).

A wide variety of parametric models has been considered in the literature to deal with asymmetric and heavy tailed distributions. We mention, the family of the $\alpha$-Stable distributions (Rachev and Mittnik, 2000), the skew t-Student distribution (Theodossiou, 1998; Jones and Faddy, 2003), the normal inverse Gaussian (Barndorff-Nielsen, 1997), the power exponential distribution (Töyli et al. 2002), the beta-type distributions (McDonald and Xu, 1995), the Tukey-type distributions (Fischer et al., 2007), and many others discussed, for instance, in Kleiber and Kotz (2003). The choice among these is usually a matter of analytical and numerical tractability. The distribution we consider possesses interesting shape characteristics, has no particular analytical and computational limitations and its cumulative distribution function, quantiles, mode and moments are given in a closed form. Moreover, the computational aspects concerning the maximum likelihood (ML) estimates do not involve great complexity. These reasons should make the log-Dagum distribution a competitive model for data marked by skewness and kurtosis.

Moreover, there is a certain analogy between log-Dagum and some other well-known distributions. For instance, its density may be considered as a reparameterization of the type I generalized logistic distribution (see Balakrishnan, 1992) and, thus, as a special case of the exponential generalized beta distribution of second type (McDonald and $\mathrm{Xu}, 1995$ ).

A random variable $X$ is log-Dagum distributed, $X \sim L D a(\beta, \lambda, \nu)$, if its distribution function is

$$
F_{X}(x ; \beta, \lambda, \nu)=\left(1+\lambda e^{-\nu x}\right)^{-\beta}
$$


where $x \in \mathbb{R}$ and $\beta, \lambda, \nu>0$. The probability density function is

$$
f_{X}(x ; \beta, \lambda, \nu)=\beta \lambda \nu e^{-\nu x}\left(1+\lambda e^{-\nu x}\right)^{-\beta-1} .
$$

From (6) it is easy to verify that the mode of the distribution is $m=\nu^{-1} \ln (\lambda \beta)$, always exists and is unique. Moreover, by solving equation $F_{X}(x ; \beta, \lambda, \nu)=p$ with respect to $x$, we obtain the simple and closed expression for the $p$-th quantile

$$
x_{p}=\frac{1}{\nu} \ln \left(\frac{\lambda}{p^{-\frac{1}{\beta}}-1}\right) .
$$

The first three moments, that will be useful in $\S 4.3$, are

$$
\begin{gathered}
E(X)=\frac{\ln (\lambda)+\Psi(\beta)-\Psi(1)}{\nu} \\
E\left(X^{2}\right)=\frac{\left[\Psi^{\prime}(\beta)+\Psi^{\prime}(1)\right]+[\ln (\lambda)+\Psi(\beta)-\Psi(1)]^{2}}{\nu^{2}} \\
E\left(X^{3}\right)=\frac{\left[\Psi^{\prime \prime}(\beta)-\Psi^{\prime \prime}(1)\right]+3[\ln (\lambda)+\Psi(\beta)-\Psi(1)]\left[\Psi^{\prime}(\beta)+\Psi^{\prime}(1)\right]}{\nu^{3}} \\
+\frac{[\ln (\lambda)+\Psi(\beta)-\Psi(1)]^{3}}{\nu^{3}}
\end{gathered}
$$

where $\Psi(),. \Psi^{\prime}(),. \Psi^{\prime \prime}($.$) are the digamma, trigamma and tetragamma functions, respectively (see,$ e.g., Davis, 1970). After simple algebra, the standardized third and fourth moments are given, respectively, by

$$
\begin{aligned}
& E\left(Z^{3}\right)=\frac{\left[\Psi^{\prime \prime}(\beta)-\Psi^{\prime \prime}(1)\right]}{\nu_{\beta}^{3}} \\
& E\left(Z^{4}\right)=\frac{\left[\Psi^{\prime \prime \prime}(\beta)+\Psi^{\prime \prime \prime}(1)\right]}{\nu_{\beta}^{4}}+3
\end{aligned}
$$

where $\nu_{\beta}=\sqrt{\Psi^{\prime}(\beta)+\Psi^{\prime}(1)}$ and $\Psi^{\prime \prime \prime}($.$) is the pentagamma function. We observe that E\left(Z^{4}\right)$ is always greater than 3 because $\Psi^{\prime \prime \prime}(\beta)>0$, for any $\beta>0$. Consequently, the log-Dagum distribution always turns out to be leptokurtic and, thus, it can be used to model data with fat tails.

\section{Maximum Likelihood Estimation}

Fitting the BB7 copula model to the Markov process $\left\{Y_{t}: t=1,2, \ldots, T\right\}$, with $Y_{t} \sim L D a(\beta, \lambda, \nu)$, requires the estimation of the marginal and association parameters. From the different ML estimation 
procedures for copulas we have adopted the full maximum likelihood method in which the marginal and association parameters are estimated simultaneously.

Given the dependent observations $y_{1}, \ldots, y_{T}$, the log-likelihood function based on the transition density (5) is

$$
\ell(\boldsymbol{\alpha} ; \boldsymbol{\xi})=\sum_{t=1}^{T} \ell_{M a r, t}(\boldsymbol{\alpha})+\sum_{t=2}^{T} \ell_{C o n, t}(\boldsymbol{\alpha} ; \boldsymbol{\xi})
$$

where

$$
\ell_{\text {Mar, } t}(\boldsymbol{\alpha})=\ln f\left(y_{t} ; \boldsymbol{\alpha}\right)=\ln (\beta \lambda \nu)-\nu y_{t}-(\beta+1) \ln \left(1+\lambda e^{-\nu y_{t}}\right)
$$

and

$$
\begin{aligned}
\ell_{C o n, t}(\boldsymbol{\alpha} ; \boldsymbol{\xi})= & \ln c\left(F\left(y_{t-1} ; \boldsymbol{\alpha}\right), F\left(y_{t} ; \boldsymbol{\alpha}\right) ; \boldsymbol{\xi}\right) \\
= & (\theta-1) \ln \bar{u}-(\delta+1) \ln \left(1-\bar{u}^{\theta}\right)+(\theta-1) \ln \bar{v}-(\delta+1) \ln \left(1-\bar{v}^{\theta}\right) \\
& +\left(\frac{1}{\theta}-2\right) \ln \left(1-\omega^{-\frac{1}{\delta}}\right)-\left(\frac{2}{\delta}+2\right) \ln \omega+\ln \left[(\theta-1)+\theta(\delta+1)\left(\omega^{\frac{1}{\delta}}-1\right)\right]
\end{aligned}
$$

with marginal and association parameters $\boldsymbol{\alpha}=(\beta, \lambda, \nu)$ and $\boldsymbol{\xi}=(\delta, \theta)$.

Maximization of (10) subject to the constraints $\beta, \lambda, \nu, \delta>0$ and $\theta>1$ leads to the ML estimates of all the parameters of the conditional approach. This optimization problem does not admit any explicit solution and requires numerical procedures which could well be computationally intensive. Under regularity conditions, the ML estimates of $\boldsymbol{\alpha}$ and $\boldsymbol{\xi}$ are consistent and asymptotically normally distributed. Moreover, the negative inverse Hessian matrix of (10) evaluated at the ML estimates can be used as an estimated covariance matrix of the ML estimators (Joe, 1997).

On the other hand, the maximization of $\sum_{t=1}^{T} \ell_{M a r, t}(\boldsymbol{\alpha})$ under constraints yields the ML estimates of parameters $\boldsymbol{\alpha}$ of the marginal approach.

Common dependence measures are usually expressed as function, say $\phi($.$) , of association param-$ eters $\boldsymbol{\xi}$. ML theory provides us with useful results concerning the estimation of the dependence measures. In particular, under usual regularity conditions, the asymptotic properties of the ML method ensure that

$$
\sqrt{n}[\phi(\hat{\boldsymbol{\xi}})-\phi(\boldsymbol{\xi})] \longrightarrow N\left(\mathbf{0}, \boldsymbol{\Sigma}_{\boldsymbol{\xi}}\right)
$$

where $\hat{\boldsymbol{\xi}}$ is the ML estimates of $\boldsymbol{\xi}$ and

$$
\boldsymbol{\Sigma}_{\boldsymbol{\xi}}=\left[\frac{\partial \phi(\boldsymbol{\xi})}{\partial \boldsymbol{\xi}}\right]^{\prime} I^{-1}(\boldsymbol{\xi})\left[\frac{\partial \phi(\boldsymbol{\xi})}{\partial \boldsymbol{\xi}}\right]
$$

with $I(\boldsymbol{\xi})$ the Fisher information matrix in a single observation. 


\section{Simulation Study}

A simulation study is now carried out to assess the ML estimation procedure and to investigate, for a finite sample size, the behavior of the ML estimates. The main idea is to generate $K$ independent samples of $T$ observations from a first order Markov process modelled by the BB7 copula and with log-Dagum margins. For each sample the ML estimates of both the association and marginal parameters are computed and an empirical version of the mean square error (MSE) is provided.

In this section, the algorithm for evaluating the performance of ML estimates of the association and marginal parameters is described first and, then, data generation and the choice of starting values are discussed in detail. Final simulation results complete the section.

\subsection{Estimation}

As the likelihood function cannot be maximized analytically, the ML estimates of the parameters are found by numerical methods involving the starting points mentioned in $\S 4.3$ as initial estimates in the optimization routine. We implemented in MATLAB a procedure based on a sequential quadratic programming method. With this method, the function adopted solves a quadratic programming subproblem at each iteration. An estimate of the Hessian of the log-likelihood is updated at each iteration using the BFGS formula.

The procedure for assessing the performance of ML estimates is based on the following steps:

1. choose real values of parameters $\beta, \lambda, \nu, \theta, \delta$ which specify the marginal distribution and the copula;

2. establish sample size $T$;

3. generate $K$ random samples of data vectors $\left(\mathbf{y}_{\mathbf{t}-\mathbf{1}}, \mathbf{y}_{\mathbf{t}}\right)$ of size $T$ following the procedure illustrated in $\S 4.2$;

4. determine the initial values, $\beta_{0}, \lambda_{0}$ and $\nu_{0}$, for the marginal parameters and $\theta_{0}, \delta_{0}$, for the association parameters, according to the criteria described in $\S 4.3$;

5. calculate, for the $k$-th of the $K$ samples of size $T$, the ML estimates $\hat{\beta}_{T}^{(k)}, \hat{\lambda}_{T}^{(k)}, \hat{\nu}_{T}^{(k)}, \hat{\theta}_{T}^{(k)}, \hat{\delta}_{T}^{(k)}$;

6. compute the mean of the estimates over all $K$ samples, $\mu\left(\hat{\zeta}_{T}\right)=K^{-1} \sum_{k=1}^{K} \hat{\zeta}_{T}^{(k)}$ where $\hat{\zeta}_{T}^{(k)}$ stands for each element of the vector $\left(\hat{\beta}_{T}^{(k)}, \hat{\lambda}_{T}^{(k)}, \hat{\nu}_{T}^{(k)}, \hat{\theta}_{T}^{(k)}, \hat{\delta}_{T}^{(k)}\right)$ for the corresponding parameter $\zeta$ among $\beta, \lambda, \nu, \theta, \delta$; 
7. determine the sample bias, $b\left(\hat{\zeta}_{T}\right)$, and the sample mean square error, $M S E\left(\hat{\zeta}_{T}\right)$, of the ML estimates calculated at the previous step

$$
b\left(\hat{\zeta}_{T}\right)=\mu\left(\hat{\zeta}_{T}\right)-\zeta, \quad M S E\left(\hat{\zeta}_{T}\right)=K^{-1} \sum_{k=1}^{K}\left(\hat{\zeta}_{T}^{(k)}-\zeta\right)^{2} .
$$

\subsection{How to simulate the data}

To generate the data we use the algorithm based on the conditional BB7 copula distribution and the quantiles of the log-Dagum marginal distribution.

Pairs of consecutive data from a first order Markov process may be thought of as observations of a pair of variables $\left(Y_{t-1}, Y_{t}\right)$ with joint distribution $F$. We are assuming that $F\left(y_{t-1}, y_{t}\right)$ is defined through the two-parameter BB7 copula function $C(u, v ; \theta, \delta)$ with margins $L D a(\beta, \lambda, \nu)$. Thus, we only need to find a suitable sequence of $(u, v)$, for each fixed value of the parameters vector $(\beta, \lambda, \nu, \theta, \delta)$, in order to obtain the corresponding pairs of observations $\left(y_{t-1}, y_{t}\right)$. The data generation algorithm works as follows:

step 1: generate a random number, say $u_{1}$, from a Uniform $(0,1)$ such that, for any $v$, $0 \leq C_{2 \mid 1}\left(v \mid u_{1} ; \theta, \delta\right) \leq 1$

step 2: iterate the following procedure $T+1$ times: generate $w_{i}$ from a Uniform $(0,1)$ and compute $v_{i}$ as the (numerical) solution of the equation $C_{2 \mid 1}\left(v \mid u_{i} ; \theta, \delta\right)=w_{i}$ where $u_{i}=v_{i-1}, i=$ $1, \ldots, T+1$, but $u_{1}$ defined at step 1 ;

step 3: the simulated data are the pair of vectors $\left(\mathbf{y}_{\mathbf{t}-1}, \mathbf{y}_{\mathbf{t}}\right)$ with $i$-th element

$$
y_{t-1}^{(i)}=\frac{1}{\nu} \ln \left(\frac{\lambda}{u_{i}^{-\frac{1}{\beta}}-1}\right), \quad y_{t}^{(i)}=\frac{1}{\nu} \ln \left(\frac{\lambda}{v_{i+1}^{-\frac{1}{\beta}}-1}\right), \quad i=1, \ldots, T .
$$

\subsection{How to choose starting values}

The choice of suitable starting points is an important issue in all numerical optimization algorithms. In this context, we adopt two distinct procedures to choose the initial values for the marginal and association parameters in order to encompass their different meanings.

Marginal parameters. Appropriate starting values for marginal parameters, $\beta, \lambda$ and $\nu$, are obtained by the method of moments equating the first three sample moments to the corresponding three moments of the log-Dagum distribution, given in (7)-(9), and solving the equations with respect to the unknowns. 
Let $\widetilde{M}_{r}$ be the sample moment of order $r$ of $Y_{t}$. Firstly, we obtain an estimate of $\beta$, say $\beta_{0}$, solving numerically the equation

$$
\frac{\Psi^{\prime \prime}(\beta)-\Psi^{\prime \prime}(1)}{\left[\Psi^{\prime}(\beta)+\Psi^{\prime}(1)\right]^{3 / 2}}=\frac{\widetilde{M_{3}}}{S^{3}}-3 \frac{\widetilde{M_{1}}}{S}
$$

being $S=\sqrt{\widetilde{M}_{2}-\widetilde{M}_{1}^{2}}$.

Afterwards, the preliminary estimates for $\lambda$ and $\nu$ are given as functions of $\beta_{0}$

$$
\lambda_{0}=\exp \left\{\widetilde{M}_{1} \sqrt{\frac{\Psi^{\prime}\left(\beta_{0}\right)+\Psi^{\prime}(1)}{S^{2}}}-\Psi\left(\beta_{0}\right)+\Psi(1)\right\}, \quad \nu_{0}=\sqrt{\frac{\Psi^{\prime}\left(\beta_{0}\right)+\Psi^{\prime}(1)}{S^{2}}} .
$$

Association parameters. In order to find a suitable pair of starting points for the association parameters, we perform a procedure which minimizes the difference between the medial correlation coefficient and an empirical version of it. Let $\mathcal{M}_{t}, \widetilde{\mathcal{M}}_{t}$ and $\mathcal{M}_{t-1}, \widetilde{\mathcal{M}}_{t-1}$ denote the population and the sample medians of $Y_{t}$ and $Y_{t-1}$, respectively. The measure of dependence mentioned in $\S 2.1$ and $\S 2.2$, now referred to the pair $\left(Y_{t-1}, Y_{t}\right)$

$$
\mathcal{M}_{t-1, t}(\theta, \delta)=P\left[\left(Y_{t}-\mathcal{M}_{t}\right)\left(Y_{t-1}-\mathcal{M}_{t-1}\right)>0\right]-P\left[\left(Y_{t}-\mathcal{M}_{t}\right)\left(Y_{t-1}-\mathcal{M}_{t}\right)<0\right]
$$

is compared with its sample version

$$
\widetilde{\mathcal{M}}_{t-1, t}=\frac{2 c-T}{T}
$$

where $c$ indicates the number of positive products of the sample median deviations, say the elementwise products of $\mathbf{y}_{\mathbf{t}}-1 \widetilde{\mathcal{M}}_{t}$ and $\mathbf{y}_{\mathrm{t}-1}-1 \widetilde{\mathcal{M}}_{t-1}$, where 1 is a column vector of ones of conformable size.

Note that we only consider data with positive medial correlation. The starting association parameters are the pair $\left(\theta_{0}, \delta_{0}\right)$ which solves the optimization problem

$$
\min _{\theta, \delta}\left(\mathcal{M}_{t-1, t}(\theta, \delta)-\widetilde{\mathcal{M}}_{t-1, t}\right) \quad \text { subject to } \quad \theta \geq 1, \delta>0
$$

\subsection{Simulation results}

We restricted the simulation study to a few combinations of values of the parameters: $\beta=0.5,1.5, \lambda=1, \nu=100, \theta=1.1,1.5$ and $\delta=0.2,1$. Actually, we considered many other values for the parameters, but the results were not significantly different from those obtained with the aforementioned values. The choice of the selected values is mainly motivated by their representativeness of a wide range of ML estimates obtained in a preliminary study in which the conditional approach was fitted to different financial data. 
Table 1: ML estimates for the parameters, bias and MSE assuming that $\lambda=1$ and $\nu=100$.

\begin{tabular}{|c|c|c|c|c|c|c|c|c|c|c|c|c|c|c|c|c|c|c|}
\hline \multirow[b]{2}{*}{$\beta$} & \multirow[b]{2}{*}{$\theta$} & \multirow[b]{2}{*}{$\delta$} & \multirow[b]{2}{*}{$n$} & \multirow[b]{2}{*}{$\hat{\beta}$} & \multirow[b]{2}{*}{$\hat{\lambda}$} & \multirow[b]{2}{*}{$\hat{\nu}$} & & & \multicolumn{5}{|c|}{ bias } & \multicolumn{5}{|c|}{ MSE } \\
\hline & & & & & & & & & $\hat{\beta}$ & $\hat{\lambda}$ & $\hat{\nu}$ & $\hat{\theta}$ & $\hat{\delta}$ & $\hat{\beta}$ & $\hat{\lambda}$ & $\hat{\nu}$ & $\hat{\theta}$ & $\hat{\delta}$ \\
\hline \multirow[t]{20}{*}{0.5} & 1.1 & 0.2 & 25 & 0.6932 & 1.3568 & 116.4598 & 1.12210 & 0.1864 & 0.1932 & 0.3568 & 16.4598 & 0.0221 & -0.0136 & 0.4727 & 2.1222 & 2783.5321 & 0.0477 & 0.0692 \\
\hline & & & 100 & 0.5452 & 1.1858 & 103.5930 & 1.1044 & 0.1898 & 0.0452 & 0.1858 & 3.5930 & 0.0044 & -0.0102 & 0.0440 & 0.7962 & 515.0156 & 0.0124 & 0.0225 \\
\hline & & & 250 & 5151 & 1.0710 & 02.2144 & 1.1010 & 0.1953 & 0.0151 & 0.0710 & 2.2144 & 0.0010 & -0.0047 & 0.0153 & 0.2518 & 220.6872 & 0.0061 & 0.0105 \\
\hline & & & 500 & 0.5052 & 1.0427 & 101.3600 & 1.0982 & 0.1984 & 0.0052 & 0.0427 & 1.3607 & -0.0018 & -0.0016 & 0.0065 & 0.0807 & 89.3769 & 0.0033 & 0.0055 \\
\hline & & & 1500 & 0.5021 & 1.0111 & 00.3500 & 1.0995 & 0.2003 & 0.0021 & 0.0111 & 0.3528 & -0.0005 & 0.0003 & 0.0020 & 0.0208 & 25.5916 & 0.0010 & 0.0018 \\
\hline & & 1 & 25 & 0.8420 & 1.2831 & 115.2771 & 1.2270 & 0.8224 & 0.3420 & 0.2831 & 15.2770 & 0.1270 & -0.1776 & 0.8195 & 2.6240 & 3653.1463 & & 0.8745 \\
\hline & & & 100 & 0.6076 & 1.2394 & 105.4515 & 1.1229 & 0.9307 & 0.1076 & 0.2394 & 5.4514 & 0.0229 & -0.0693 & 0.1820 & 1.1853 & 587.1129 & 0.02 & 0.1926 \\
\hline & & & 250 & .5348 & 1.0628 & 101.4600 & & 0.9765 & 0.0348 & 0.0628 & 1.4600 & 0.0096 & -0.0235 & 0.0275 & 0.2538 & 175.0398 & & \\
\hline & & & 500 & 0.5144 & 1.0375 & 101.3432 & 1.0977 & 0.9962 & 0.0144 & 0.0375 & 1.3431 & -0.0023 & -0.0038 & 0.0122 & 0.0021 & 90.0023 & 0.00 & 0.0461 \\
\hline & & & 1500 & 5051 & 1.0098 & 100.4400 & 1.0990 & 1.0013 & 0.0051 & 0.0098 & 0.4416 & -0.0010 & 0.0013 & 0.0034 & 0.0297 & 27.5113 & & 0.0128 \\
\hline & 1.5 & 0.2 & 25 & 0.6006 & & & & & & & 34.4379 & & & & & 5677.2752 & & 0.1129 \\
\hline & & & 100 & 0.5599 & 1.2796 & 107.4658 & 1.4825 & 0.2342 & 0.0599 & 0.2796 & 7.4658 & -0.0175 & 0.0342 & 0.0708 & 1.2631 & 962.1720 & 0.0 & 0.0422 \\
\hline & & & 250 & 0.5178 & 1.1039 & 103.2698 & & 0.1930 & 0.0178 & 0.1039 & 3.2698 & & -0.0070 & 0.0218 & & 328.2868 & & 0.0157 \\
\hline & & & 500 & 0.5109 & 1.0415 & 101.5400 & 1.5013 & 0.1965 & 0.0109 & 0.0415 & 1.5439 & 0.0013 & -0.0036 & 0.0090 & 0.0983 & 144.3747 & & 0.0077 \\
\hline & & & 1500 & 5041 & 1.0018 & 100.5100 & $1.4 \mathrm{C}$ & 0.1987 & 0.0041 & 0.0018 & 0.5146 & 007 & & 0.0026 & & 43.4389 & & 0.0026 \\
\hline & & 1 & 25 & 0.7468 & 1.5045 & 129.2929 & 1.4857 & 0.9198 & 0.2468 & 0.5045 & 29.2929 & -0.0143 & -0.0 & 0.6468 & 3.2669 & 5221.6529 & & 1.1405 \\
\hline & & & 100 & 0.6539 & 1.3485 & 107.6083 & & 0.9717 & 0.1539 & 0.3485 & 7.6083 & & -0.02 & 0.2768 & & 1223 & & 0.3964 \\
\hline & & & 250 & & & & & & & & & & & & & & & \\
\hline & & & 500 & 0.5327 & 1.0398 & 100.8700 & 1.5080 & 0.9810 & 0.0327 & 0.0398 & 0.8722 & 0.0080 & -0.0190 & 0.0208 & 0.1808 & 185.6391 & & 0.0554 \\
\hline & & & 1500 & 5069 & 1.0142 & 100.67 & 1.4993 & 0.9944 & 0069 & .0142 & 791 & 007 & -0.0056 & 0.0050 & 420 & 54.2930 & & 160 \\
\hline \multirow[t]{20}{*}{1.5} & & 0.2 & 25 & 2.8999 & 1.7446 & 114.0950 & 1.1303 & 0.2347 & 1.3999 & 0.7446 & 14.0950 & 0.0303 & & 13.0351 & 4.2341 & 1243.4969 & & 899 \\
\hline & & & 100 & 2.0770 & 1.1908 & 101.7093 & 1.1060 & 0.1899 & 0.5770 & 0.1908 & 1.7093 & 0.0060 & 0101 & 3.0804 & 1.0441 & 294.2359 & & 225 \\
\hline & & & 250 & 1.6833 & 1.0693 & 101.1691 & 1.1021 & 0.1938 & 0.1833 & 0.0693 & 1.1691 & & -0.00 & 0.5081 & 0.2968 & 120.1334 & & 105 \\
\hline & & & 500 & 1.5742 & 1.0461 & 100.7500 & 1.1024 & 0.1918 & 0.0742 & 0.0461 & 0.7500 & 0.0024 & -0.0082 & 0.1631 & 180 & 55.9578 & & 0054 \\
\hline & & & 1500 & & 1.0093 & 100.2800 & 1.0999 & & 0.0258 & & 0.2773 & -0.0001 & & & & 16.2936 & & 0017 \\
\hline & & 1 & 25 & 3.5305 & 1.3960 & 111.0139 & 1.2073 & 0.9059 & 2.0305 & 0.3960 & 11.0139 & 0.1073 & -0.0941 & 17.7557 & 3.1168 & 1082.5054 & & 0.9021 \\
\hline & & & 100 & 2.3996 & 1.1008 & & 1.1177 & 0.9007 & 0.8996 & & & & & & & & & 134 \\
\hline & & & 250 & 1.8504 & 1.0249 & 100.3605 & 1.1069 & 0.9842 & 0.3504 & 0.0249 & 0.3605 & 0.0069 & -0.0158 & 1.3020 & 0.3120 & 99.2082 & & 0.0826 \\
\hline & & & 500 & 1.6426 & 1.0167 & 100.4500 & 1.1035 & 0.9860 & 0.1426 & 0.0167 & 0.4481 & 0.0035 & -0.0140 & 0.2975 & 0.1348 & 52.4535 & 0.0053 & 0.0427 \\
\hline & & & 1500 & 1.5479 & 0.9974 & 100.2500 & 1.0994 & 0.9907 & 0.0479 & -0.0026 & 0.2477 & -0.0006 & -0.0093 & 0.0565 & 0.0397 & 16.7592 & & 0.0128 \\
\hline & 1.5 & 0.2 & 25 & 2.4618 & 2.1271 & 123.4661 & 1.3801 & 0.2422 & 0.9618 & 1.1271 & 23.4661 & -0.1199 & 0.0422 & 9.8025 & 5.8040 & 1922.7497 & 0.1 & 0.1125 \\
\hline & & & 100 & 2.1517 & 1.3402 & & 1.4957 & & 0.6517 & & 3.7283 & & & & & 527.6009 & & 315 \\
\hline & & & 250 & 1.7484 & 1.1031 & 101.9046 & 1.4986 & 0.1946 & 0.2484 & 0.1031 & 1.9046 & -0.0014 & -0.0054 & 0.9282 & 0.4026 & 210.5594 & 0.0320 & 0.0154 \\
\hline & & & 500 & & 1.0406 & 100.5600 & 1.5078 & 0.1946 & 0.1178 & 0.0406 & 0.5594 & 0.0078 & -0.0054 & 0.2415 & 0.1619 & 96.2569 & 0.0 & 0.0072 \\
\hline & & & 1500 & 1.5466 & 0.9970 & 99.9290 & 1.5051 & 0.1990 & 0.0466 & -0.0030 & -0.0706 & 0.0051 & -0.0011 & 0.0524 & 0.0404 & 29.3305 & 0.00 & 0.0024 \\
\hline & & 1 & 25 & 3.0958 & 1.7938 & 124.5383 & 1.3931 & 0.8660 & 1.5958 & 0.7938 & 24.5383 & -0.1069 & -0.1340 & 14.3620 & 4.8516 & 2323.6832 & 0.245 & 0.8617 \\
\hline & & & 100 & 2.1561 & 1.4806 & 107.7377 & 1.4494 & 0.9324 & 0.6561 & 0.4806 & & -0.0506 & -0.0676 & & 2.1766 & 516.8924 & 0.08 & 0.3666 \\
\hline & & & 250 & 2.0162 & 1.0864 & 101.0619 & 1.5061 & 0.9905 & 0.5162 & 0.0864 & 1.0619 & 0.0061 & -0.0095 & 2.7565 & 0.5607 & 196.2678 & 0.0432 & 0.1112 \\
\hline & & & 500 & 1.6641 & 1.0511 & 101.0100 & 1.5004 & 0.9925 & 0.1641 & 0.0511 & 1.0051 & 0.0004 & -0.0075 & 0.4136 & 0.2147 & 94.9761 & 0.01 & 0.0548 \\
\hline & & & 1500 & 1.5897 & 0.9763 & 99.7510 & 1.5084 & 0.9844 & 0.0897 & -0.0237 & -0.2492 & 0.0084 & -0.0156 & 0.0925 & 0.0550 & 30.6906 & 0.0072 & 0.0173 \\
\hline
\end{tabular}


Since the estimation procedure of both marginal and association parameters may be of great computational complexity, the simulation study has been performed with each combination of selected parameters for $K=1000$ samples with increasing size $T=25,100,250,500,1500$.

The study allowed us to attain two goals: to check the reliability of our estimation procedure and to investigate the behavior of the estimators for a finite sample size. The results, summarized in Table 1, confirm the good performance of the estimators. As expected, the performance is modest for smaller sample sizes but improves remarkably as the sample size increases. In a few cases we observe an oscillatory behavior of the bias, that is, it registers decreases followed by slight nonregular increases. Nevertheless, the mean square error seems to approach very small values and certainly decreases as the sample size rises, an evident indication that all the estimates are consistent.

\section{Empirical Analysis}

The conditional approach is now applied to real data in order to go beyond the normality and i.i.d. hypotheses traditionally assumed for the distribution of financial returns. Firstly, we highlight certain stylized facts that mark consecutive financial returns $Y_{t}$ and $Y_{t-1}$. In particular, through an empirical study, we note evidence of non-normality in the returns, as well as the presence of dependence. Then, we model the data with the conditional and the marginal approach showing how the use of the copula may considerably improve the fitting to data when dependence between consecutive returns is ascertained.

\subsection{Departure from normality and independence}

For our analysis we consider the daily returns of four Italian stocks: Generali from January 8th, 1999 to January 7th, 2004; Banca Popolare Italiana (Bpi) from January 2nd, 1995 to August 7th, 2006; Telecom from January 8th, 1999 to January 7th, 2004; Tiscali from October 27th, 1999 to January 19th, 2005.

The descriptive statistics given in Table 2 highlight that the returns are negatively or positively skewed with a rather high level of kurtosis for Tiscali and Bpi stocks. The Normal assumption for describing the data seems to be inadequate and this is confirmed by the value of the Jarque-Bera statistic test and the QQ-plot in Figure 2. The QQ-plot in Figure 3, instead, shows that the log-Dagum distribution strongly fits the empirical distribution of Telecom and Generali series, whereas it is less suitable for Bpi and Tiscali. Such behavior may be referable to the fact that the latter two series are characterized either by high kurtosis and, as we will see later, strong evidence of temporal dependence between consecutive returns which is indeed underrated in this marginal approach. 
Table 2: Descriptive statistics for daily returns.

\begin{tabular}{lcccc}
\hline & Generali & Bpi & Telecom & Tiscali \\
\hline mean & -0.0005 & 0.0002 & -0.0006 & -0.0008 \\
standard deviation & 0.0192 & 0.0171 & 0.0280 & 0.0386 \\
skewness & -0.0351 & 0.0600 & -0.2324 & 1.2079 \\
kurtosis & 5.0013 & 20.759 & 5.9827 & 11.005 \\
JB Test & 212.12 & 38530 & 478.93 & 4099.8 \\
\hline
\end{tabular}

Generali

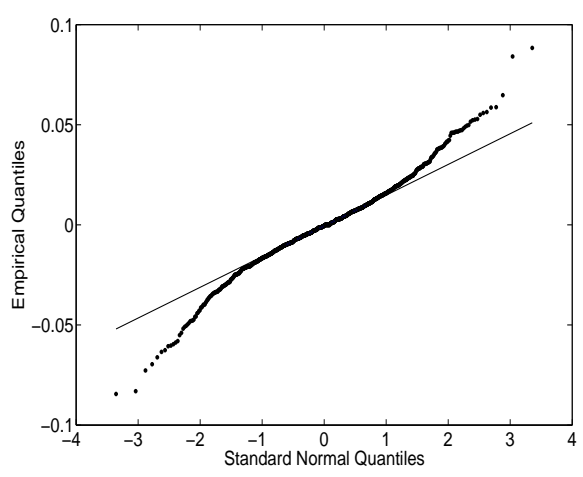

Tiscali

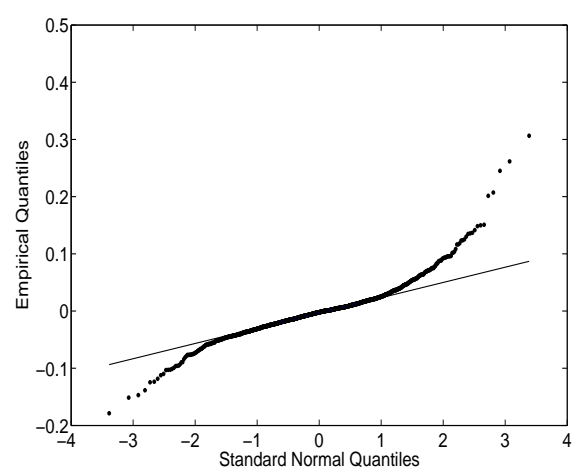

Telecom

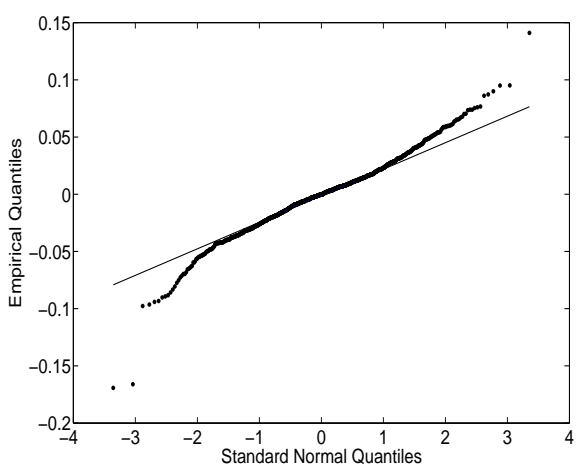

Bpi

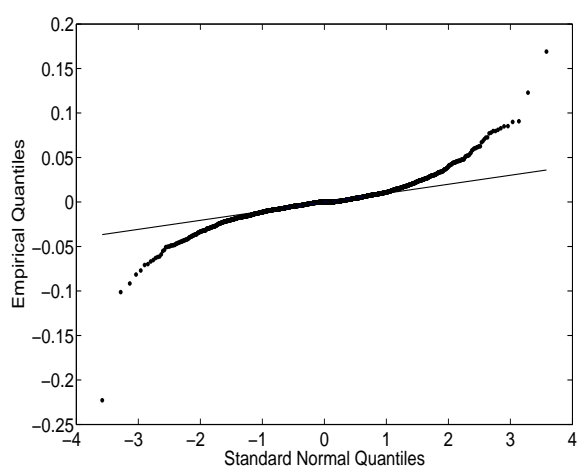

Figure 2: Normal QQ-plot for daily returns.

The typical i.i.d. hypothesis underlying most common statistical procedures is to be treated with great caution when modelling financial returns. Many authors have studied the dependence between two or more financial assets. Here, our main intention is to investigate the temporal dependence for a financial time series.

It is well-known (see Fama, 1971; Pagan 1996) that movements of returns in liquid markets do not exhibit significant correlation. The lack of correlation has been widely discussed and is often cited as 


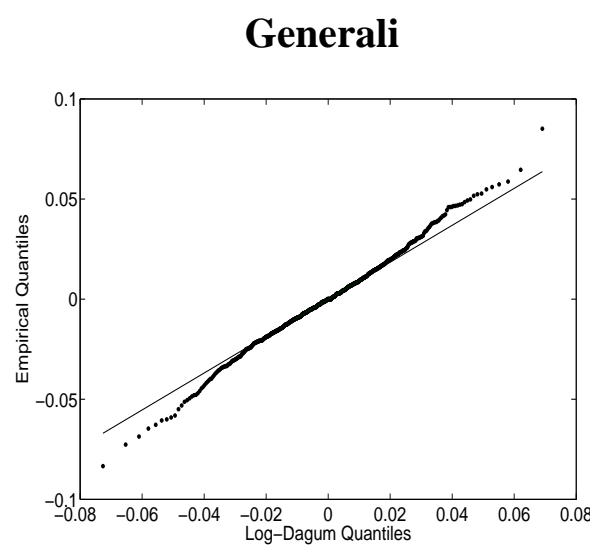

Telecom

Tiscali
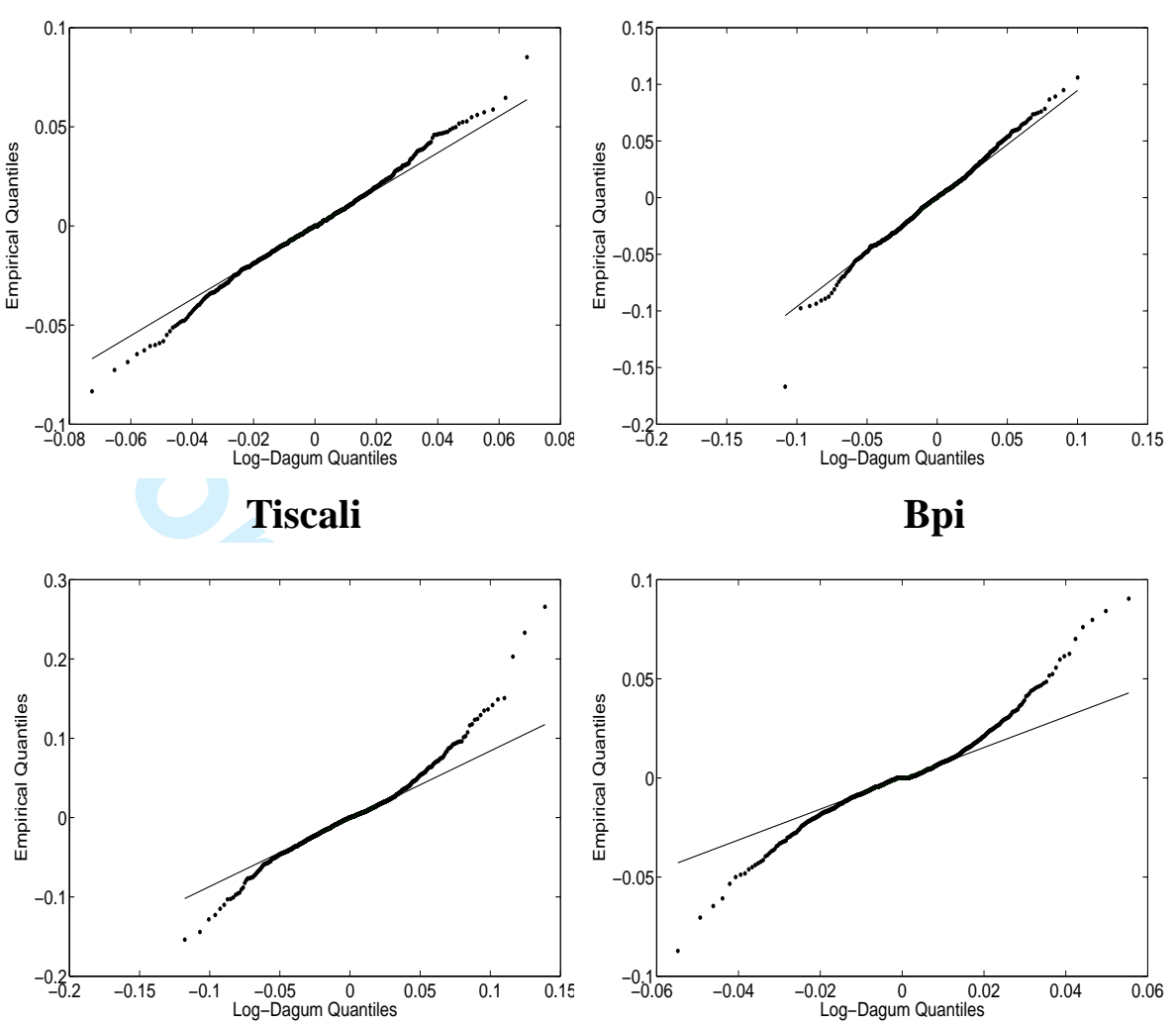

Bpi

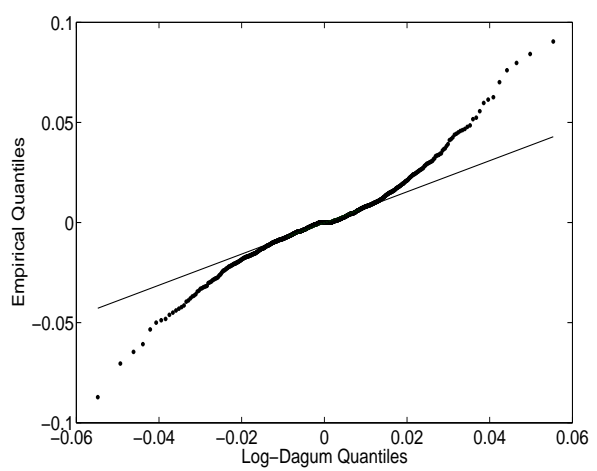

Figure 3: Log-Dagum QQ-plot for daily returns.

support for the efficient market hypothesis (Fama, 1991). Nevertheless, uncorrelation does not mean that the return movements are independent. Other forms of nonlinear dependence may exist which cannot be conveyed by the traditional dependence measures.

Common measures of nonlinear dependence are based on the autocorrelation of various powers of the absolute returns

$$
\rho_{[r]}=\operatorname{corr}\left(\left|Y_{t-1}\right|^{r},\left|Y_{t}\right|^{r}\right) .
$$

Typical choices for $r$ are 1 and 2. For $r=1$, the correlation is the highest, which means that the absolute returns are more predictable than other powers of returns. The choice $r=2$ provides a measure of the phenomenon of volatility clustering or volatility-volatility correlation: large/small return variations are more likely to be followed by large/small return variations. Figure 4 shows this phenomenon for the daily returns of the considered stocks. 


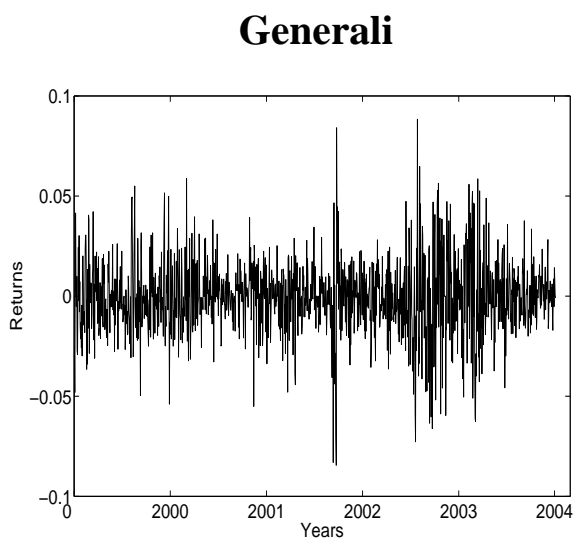

Tiscali

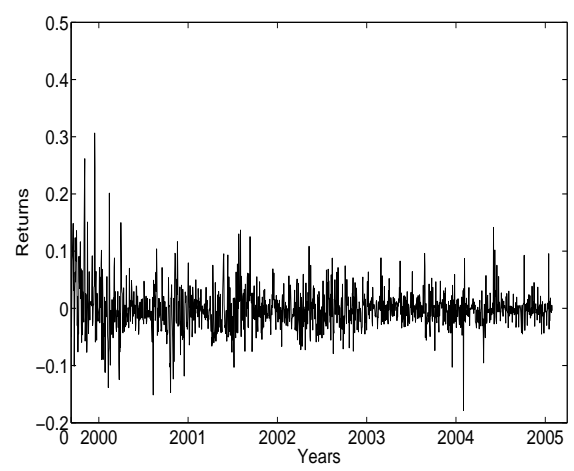

Telecom

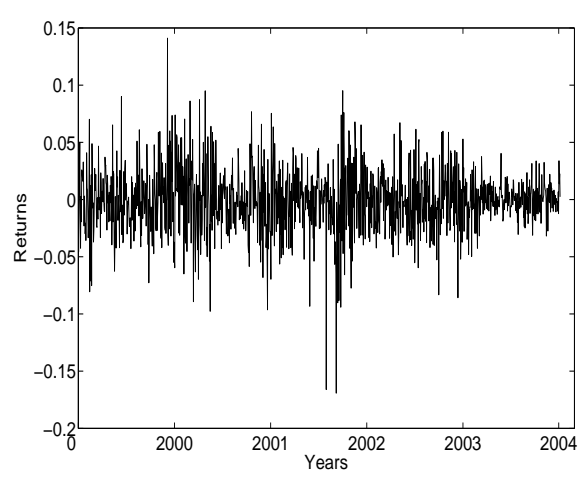

Bpi

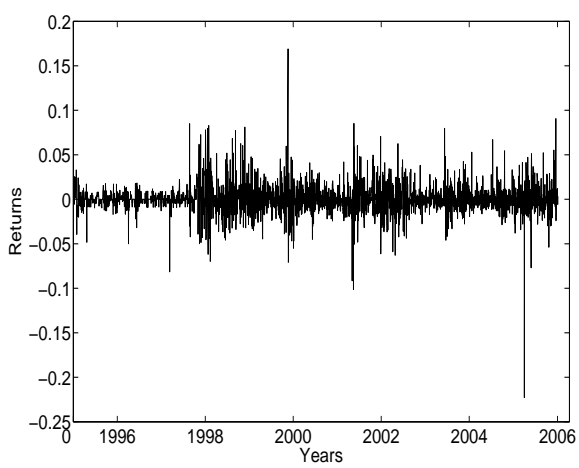

Figure 4: Daily returns and volatility cluster phenomenon.

\subsubsection{Graphical assessment of dependence: the chi-plot}

The chi-plot proposed by Fisher and Switzer $(1985,2001)$ is a ranked-based graphical tool that reveals more detailed and explicit information regarding the nature of dependence between two variables. In particular it has a characteristic pattern depending on whether the variables (i) are independent, (ii) have some degree of monotone relationship, (iii) have a more complex dependence structure.

For our aim, let us consider the bivariate variable $\left(Y_{t-1}, Y_{t}\right)$ and let $\left(y_{t-1}^{(i)}, y_{t}^{(i)}\right)$ be the $i$-th data point $(i=1, \ldots, T)$ from the vectors of sample observations $\left(\mathbf{y}_{\mathbf{t}-\mathbf{1}}, \mathbf{y}_{\mathbf{t}}\right)$. Moreover, let $I(A)$ be the indicator function taking values 0 or 1 according as $A$ is true or false. For each data point $\left(y_{t-1}^{(i)}, y_{t}^{(i)}\right)$ set

$$
\begin{gathered}
Z_{i}=\frac{1}{T-1} \sum_{j \neq i}^{T} I\left(y_{t-1}^{(j)} \leq y_{t-1}^{(i)}, y_{t}^{(j)} \leq y_{t}^{(i)}\right) \\
W_{i}=\frac{1}{T-1} \sum_{j \neq i}^{T} I\left(y_{t-1}^{(j)} \leq y_{t-1}^{(i)}\right), \quad G_{i}=\frac{1}{T-1} \sum_{j \neq i}^{T} I\left(y_{t}^{(j)} \leq y_{t}^{(i)}\right)
\end{gathered}
$$

and

$$
S_{i}=\operatorname{sign}\left\{\left(W_{i}-0.5\right) \cdot\left(G_{i}-0.5\right)\right\}
$$


where $\operatorname{sign}(\cdot)$ denotes the sign function. Now, define the quantities

$$
\chi_{i}=\frac{Z_{i}-W_{i} G_{i}}{\sqrt{W_{i}\left(1-W_{i}\right) G_{i}\left(1-G_{i}\right)}}
$$

and

$$
\eta_{i}=4 S_{i} \max \left\{\left(W_{i}-0.5\right)^{2},\left(G_{i}-0.5\right)^{2}\right\}
$$

The chi-plot is the scatterplot of the pairs $\left(\eta_{i}, \chi_{i}\right)$. At each sample point $\left(y_{t-1}^{(i)}, y_{t}^{(i)}\right), \chi_{i} \in[-1,1]$ measures the departure from bivariate independence. If $Y_{t}$ is a strictly increasing function of $Y_{t-1}$, then $\chi_{i}=1$ for all the sample points; similarly, if $Y_{t}$ is a strictly decreasing function of $Y_{t-1}$, then $\chi_{i}=-1, i=1,2, \ldots, T$. Moreover, $\eta_{i} \in[-1,1]$ is a measure of the distance of $\left(y_{t-1}^{(i)}, y_{t}^{(i)}\right)$ from the center of the dataset as expressed by the median of $\left(\mathbf{y}_{\mathbf{t}-1}, \mathbf{y}_{\mathbf{t}}\right)$.

When the bivariate data come from independent margins, $\chi_{i}$ values tend to be uniformly concentrated in a strip around the horizontal lines $\chi=0$. The width of the strip is determined so that approximately $100 p \%$ of pairs $\left(\eta_{i}, \chi_{i}\right)$ lies between two control lines, say $\chi= \pm c_{p} / \sqrt{T}$. The $c_{p}$ values $1.54,1.78$ and 2.18 correspond to $p=0.90,0.95$ and 0.99 , respectively (Fisher and Switzer, 2001). However, when $Y_{t}$ and $Y_{t-1}$ are dependent, the values of $\eta_{i}$ show clustering structures. In particular, if $Y_{t}$ and $Y_{t-1}$ are characterized by a positive relationship, $\chi_{i}$ values will tend to be positive and the converse for negative dependence. Dependence between $Y_{t}$ and $Y_{t-1}$ should be revealed by departures from this zero-centered horizontal strip.

Recently, different uses of the chi-plot have been suggested. For instance, Genest and Boies (2003) discussed the connection between ranked marginal data and copulas. Abberger (2005) used the chiplot to explore local dependence in the tails of a bivariate distribution. He stated that lower/upper tail independence is present in the data when the chi-plot inclines on the left/right side of the graph to the zero line.

\subsection{Checking for dependence}

With the aim of assessing possible forms of dependence in the pairs $\left(Y_{t}, Y_{t-1}\right)$, an empirical study of the concerned time series is now performed. Preliminary results regarding monotone dependence (i.e. nonzero grade correlation) between $Y_{t}$ and $Y_{t-1}$ are shown in Table 3. Here we consider Kendall's tau, Spearman's rho, Pearson's correlation coefficient, the medial correlation coefficient and the measures of nonlinear dependence, $\rho_{[1]}$ and $\rho_{[2]}$, given in (11).

A slight form of monotone association emerges from the observed data even if $\rho_{[1]}$ and $\rho_{[2]}$ put more emphasis to nonlinear dependence. Nonlinear dependence is particularly evident for Tiscali, Bpi and Generali series. 
Table 3: Measures of dependence between consecutive daily returns.

\begin{tabular}{lcccccc}
\hline & $\tau$ & $\rho_{S}$ & $\rho$ & $\mathcal{M}$ & $\rho_{[1]}$ & $\rho_{[2]}$ \\
\hline \hline Generali & 0.0110 & 0.0167 & 0.0523 & 0.0174 & 0.2177 & 0.2476 \\
Telecom & 0.0365 & 0.0534 & 0.0533 & 0.0079 & 0.1199 & 0.0671 \\
Tiscali & 0.0748 & 0.1110 & 0.2155 & 0.0675 & 0.3434 & 0.3816 \\
Bpi & 0.0374 & 0.0547 & 0.1340 & 0.0338 & 0.3348 & 0.1824 \\
\hline
\end{tabular}

Generali

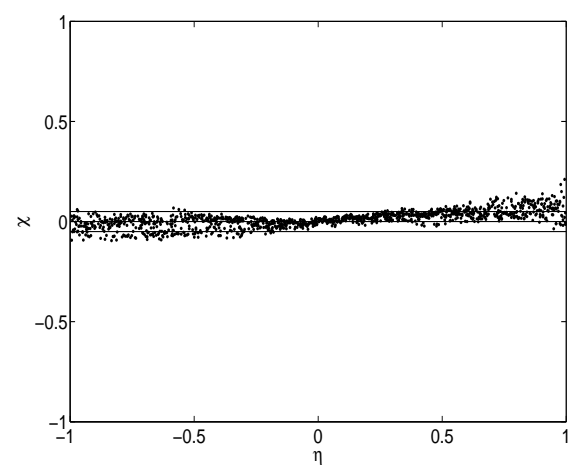

Tiscali

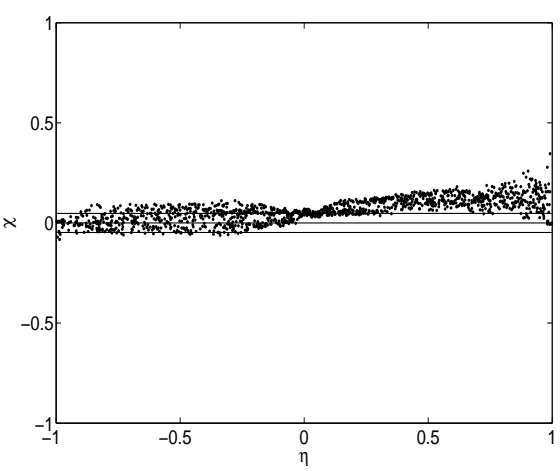

Telecom

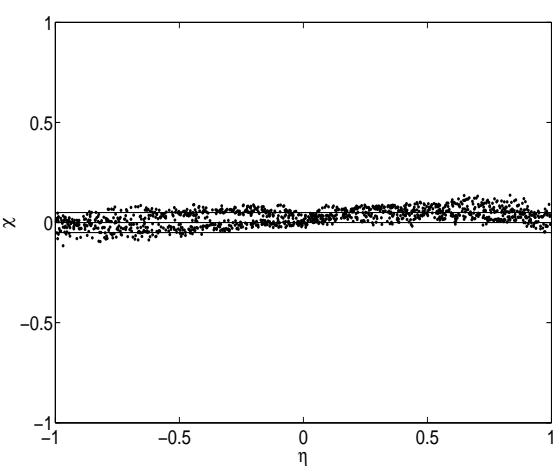

Bpi

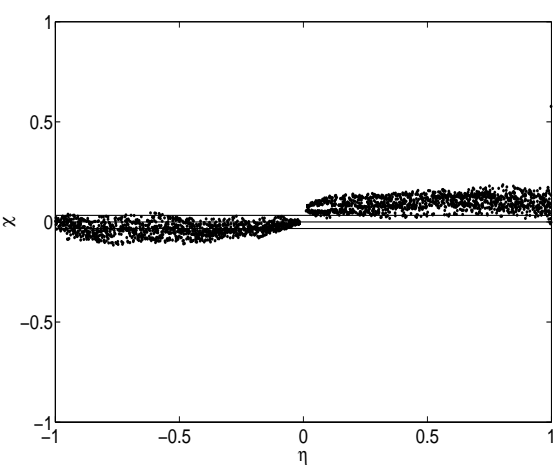

Figure 5: Chi-plot for daily returns.

To investigate the structure of the dependence underlying the series more thoroughly, we integrate the descriptive measures with the chi-plot analysis displayed in Figure 5. In general, we can observe that $\chi_{i}$ values are centered around zero, but are not uniformly distributed along $\eta$-axis: clustering structures and a certain pattern of positive and negative dependence appear. More specifically, the chi-plot for Generali series highlights the absence of any relationship between the returns for $\eta_{i}<0$, whereas positive dependence is visible for $\eta_{i}>0$. A possible form of upper tail dependence should be considered. For Telecom, we note a rather bent course for $\eta_{i}>0$ indicating a slight local positive dependence. Moreover, most $\chi_{i}$ values are positive and lie outside the upper control line. On the 
contrary, $\chi_{i}$ values seem to be reasonably uniformly distributed for $\eta_{i}<0$. No tail dependence is shown. The graph produces approximately a horizontal diagram denoting independence between the consecutive returns. The graphical analysis for Tiscali does not show any dependence structure for $\eta_{i}<0$ since $\chi_{i}$ values are uniformly concentrated around zero. On the other hand, for positive values of $\eta$, the chi-plot reveals the presence of positive dependence of a complex nature since $\chi_{i}$ values appear in terms of increased scatter outside the upper control line. In particular, for values $\eta_{i}$ near to $1, \chi_{i}$ values appear unusually high. These $\eta_{i}$ correspond to large peripheral returns which are of the same sign. The evidence of peripheral dependence seems to convey upper tail dependence. As far as the Bpi series is concerned, both positive and negative asymmetric dependencies appear. For $\eta_{i}<0$ the graph traces a bent course while for $\eta_{i}>0$ almost all $\chi_{i}$ values are scattered outside the upper control line. Upper tail dependence is detected. To conclude, we can state that the Bpi and Tiscali series are marked by a prominent dependence while Generali is characterized by a modest degree of dependence. Independence between consecutive returns seems to appear in Telecom data.

\subsection{Fitting models to the data}

Since the empirical analysis of the daily returns shows evidence of possible forms of dependence between the returns, the adoption of the copula approach appears particularly appropriate to ascertain and measure the temporal dependence between $Y_{t}$ and $Y_{t-1}$.

This section is devoted to the comparison between the marginal and conditional approach used to model the data. The former considers the returns as a sequence of i.i.d. observations from a logDagum distribution. The latter relies on the assumption that returns are generated from a first order stationary Markov process which here is modelled through a BB7 copula function with log-Dagum margins. The performance of the two approaches is compared by means of the values of the loglikelihood functions and the Akaike Information Criterion (AIC)

$$
A I C=-2 \ell+2 q
$$

where $\ell$ denotes the log-likelihood function evaluated in the ML estimates (either for the marginal and conditional approach) and $q$ is the number of parameters. Models with smaller AIC values are to be preferred to those with larger ones.

Tables 4 and 5 report the ML estimates of the parameters of marginal and conditional models together with the aforementioned indices. We preliminarily observe that marginal estimates are significant for all the series, whereas the estimates of association parameters are significant for Generali, Tiscali and Bpi. 
Table 4: ML estimates and p-value for the parameters of the marginal approach.

\begin{tabular}{l|ccc|cc}
\hline & $\beta$ & $\lambda$ & $\nu$ & $\ell$ & $A I C$ \\
\hline \hline Generali & 0.9522 & 1.0225 & 99.586 & 3247.6 & -6489.3 \\
p-value & 0.0000 & 0.0000 & 0.0000 & & \\
\hline Telecom & 0.9083 & 1.1162 & 69.155 & 2771.9 & -5537.9 \\
p-value & 0.0000 & 0.0000 & 0.0000 & & \\
\hline Tiscali & 1.3439 & 0.5917 & 48.050 & 2725.1 & -5444.3 \\
p-value & 0.0000 & 0.0000 & 0.0000 & & \\
\hline Bpi & 1.0265 & 0.9350 & 123.980 & 8162.4 & -16319 \\
p-value & 0.0000 & 0.0000 & 0.0000 & & \\
\hline \hline
\end{tabular}

Table 5: ML estimates and p-value for the parameters of the conditional approach.

\begin{tabular}{l|ccccc|cc}
\hline & $\beta$ & $\lambda$ & $\nu$ & $\theta$ & $\delta$ & $\ell$ & $A I C$ \\
\hline \hline Generali & 0.9553 & 1.0105 & 100.22 & 1.0426 & 0.0602 & 3254.2 & -6498.1 \\
p-value & 0.0000 & 0.0000 & 0.0000 & 0.0128 & 0.0138 & & \\
\hline Telecom & 0.9115 & 1.1084 & 69.101 & 1.0349 & 0.0395 & 2772.8 & -5535.5 \\
p-value & 0.0000 & 0.0000 & 0.0000 & 0.0666 & 0.0860 & & \\
\hline Tiscali & 1.2658 & 0.6222 & 50.48 & 1.0793 & 0.1164 & 2775.8 & -5541.6 \\
p-value & 0.0000 & 0.0000 & 0.0000 & $1.59 \mathrm{E}-05$ & $5.38 \mathrm{E}-05$ & & \\
\hline Bpi & 1.000 & 0.9478 & 128.90 & 1.0478 & 0.0822 & 8232.3 & -16455 \\
p-value & 0.0000 & 0.0000 & 0.0000 & $9.00 \mathrm{E}-07$ & $1.68 \mathrm{E}-07$ & & \\
\hline
\end{tabular}

Looking at AIC for Tiscali and Bpi series, it is quite evident that the conditional approach based on the BB7 copula shows a better fitting to data than the marginal approach. The conditional approach seems to hold for Generali returns as well. On the contrary, the marginal approach should be adopted for modelling Telecom series.

These results are perhaps not surprising since Tiscali and Bpi series are marked by stronger dependence than Generali and Telecom series, as shown in the previous empirical analysis. This is further confirmed by the fact that the estimates of the association parameters, $\theta$ and $\delta$, are highly significant for the Tiscali and Bpi series, significant for Generali, but not for Telecom. This means that when temporal dependence characterizes the series, an approach which allows for this aspect is to be preferred. On the other hand, when there is no dependence, it would be better to adopt a more parsimonious approach that ignores it. Thus, the marginal approach is supposed to fit better to data which show independence, and the conditional approach should be favoured otherwise, even if the series denote a low level of dependence. Our results confirm this expectation. Moreover, in the light of these considerations, the log-Dagum QQ-plot of Figure 3 should be now more informative and be explained in terms of dependence, ceteris paribus. The poor fitting of the marginal log-Dagum approach for Tiscali and Bpi series may also be ascribed to the ascertained nonnegligible temporal dependence. 
Table 6: ML estimates and p-value for medial correlation $(\mathcal{M})$ and Kendall's tau $(\tau)$.

\begin{tabular}{l|cc}
\hline & $\mathcal{M}$ & $\tau$ \\
\hline \hline Generali & 0.0485 & 0.0510 \\
p-value & 0.0002 & 0.1889 \\
\hline Telecom & 0.0358 & 0.0384 \\
p-value & 0.0007 & 0.2962 \\
\hline Tiscali & 0.0880 & 0.0939 \\
p-value & 0.0000 & 0.0430 \\
\hline Bpi & 0.0602 & 0.0642 \\
p-value & 0.0000 & 0.0187 \\
\hline
\end{tabular}

Furthermore, the QQ-plot highlights a very good marginal fitting for Telecom and Generali series where the temporal dependence is quite irrelevant. This should further strengthen the validity of the conditional approach when temporal dependence is found.

Table 6 provides the ML estimates for Kendall's tau and the medial correlation coefficient obtained by substituting in (2) and (4) the ML estimates of $\theta$ and $\delta$. The medial correlation coefficient is significantly different from zero for all the series, while Kendall's tau appears significant for Bpi and, to a lesser extent, for Tiscali. Similar conclusions may be drawn from Table 7 which reports the ML estimates of the measures $\lambda_{L}=2^{-1 / \delta}$ and $\lambda_{U}=2-2^{1 / \theta}$ discussed in $\S 2.2$. The $\lambda_{L}$ estimates are not significantly different from zero while $\lambda_{U}$ are highly significant for Tiscali and Bpi and significant for Generali, as expected from the results highlighted in $\S 5.2$.

The estimated dependence measures seem to be consistent with the previous considerations because they highlight particular forms of dependence (global or local) only for the series which exhibit marked temporal dependence.

These outcomes are very promising since they support the choice of the conditional approach for modelling the temporal dependence in univariate time series. This conclusion is not limited to the use of the BB7 copula, it is definitely more general and we expect that it holds for other choices of the copula as well. We recommend, therefore, the copula approach for situations in which non-negligible dependence is found in the observed data. In this sense, a preliminary analysis of the data may provide useful indications on the type and the level of the dependence.

\section{Final Remarks}

In many applications, the hypotheses of independence and normality of the data are usually assumed for convenience because easier to be handled. However, there are situations where neglecting dependence and non-normality effects may yield misleading models and unrealistic estimates of unknown 
parameters. The care of dependence and non-normality becomes important to extend the standard model towards more efficient ones. Nevertheless, if the previous assumptions are relaxed, much less tractable models are necessary.

This paper is an attempt to deal with temporal dependence between consecutive returns in univariate financial time series. We have assumed that the financial return at time $t-1$ influences the behavior of the return at time $t$. In this spirit, a conditional approach has been employed. Financial returns have been modelled through a first order stationary Markov process using a copula function and taking into account departure from Normal distribution. In particular, to describe the dependence structure underlying a time series we have considered the Archimedean two-parameter BB7 copula whereas, in order to capture skewness and kurtosis that mark the data, we have chosen the log-Dagum distribution. Finally, the conditional approach has been compared through empirical analysis with the marginal one. The latter relies on the independence assumption between consecutive returns which are log-Dagum distributed.

Our main findings highlight that the conditional approach performs better than the marginal one for time series which exhibit structures of temporal dependence. Models based on the conditional approach should better fit the data and provide a more reliable representation of reality. This aspect assumes particular importance, for instance, in finance where it is hard for financial institutions and regulators to avoid dramatic underestimation of appropriate risk measures. However, we observe that the results obtained in the paper are not only relevant for financial applications, but also for the analysis of any real-life time series where the assumption of independence and normality seems inappropriate.

Obviously, to understand more fully the potential of this approach, further investigations are required. Some problems are still open and need an in-depth study. For instance, it would be useful to analyze the characteristics of the conditional distribution of a variable at time $t$ given its behavior at $t-1$. At the same time, we could extend the concept of temporal dependence by considering a 
higher-order Markov process and assess the performance of our approach for forecasting analysis.

Acknowledgements. The work has been supported by Murst ex-60\% funds, University of Calabria. The authors wish to thank Prof. Ivar Massabó and two anonymous referees for their careful reading and constructive suggestions which led to improvements over an earlier version of the paper. 


\section{References}

[1] Abberger, K. (2005). A simple graphical method to explore tail-dependence in stock-return pairs. Applied Financial Economics, 15, 43-51.

[2] Balakrishnan, N. (1992). Handbook of the Logistic Distribution. Statistics: a Series of Textbooks and Monographs, New York: Marcel Dekker, Inc.

[3] Barndorff, O. E. (1997). Normal inverse Gaussian distributions and the modelling of stock returns. Scandinavian Journal of Statistics, 24, 1-13.

[4] Bingham, N. H., Schmidt, R. (2005). High-frequency data - interplay between distributional and temporal dependence. In: From Stochastic Analysis to Mathematical Finance - Festschrift for A.N. Shiryaev. Kabanov Y. and Lipster R. (Eds.), New York: Springer Verlag.

[5] Blomqvist, N. (1950). On a measure of dependence between two random variables, Annals of Mathematical Statistics, 21, 593-600.

[6] Bouyé, E., Gaussel, N., and Salomon, M. (2002). Investigating dynamic dependence using copulae. Tech. Rep., Financial Econometrics Research Center.

[7] Capéraà, P., Genest, C. (1993). Spearman's rho is larger than Kendall's tau for positively dependent random variables. Journal of Nonparametric Statistics, 2, 183-194.

[8] Chen, X., Fan, Y. (2006). Estimation of copula-based semiparametric time series models. Journal of Econometrics, 130, 307-335.

[9] Cherubini, U., Luciano, E. and Vecchiato, W. (2004). Copulas Methods in Finance. Chichester: John Wiley \& Sons.

[10] Cont, R. (2001). Empirical properties of asset returns: stylized fact and statistical issues. Quantitative Finance, 1, 223-236.

[11] Dagum, C. (1977). A new model of personal income distribution: specification and estimation. Economie Appliquée, 30, 413-437.

[12] Dagum, C. (1980). The generation and distribution of income, the Lorenz curve and the Gini ratio. Economie Appliquée, 33, 327-367.

[13] Davis, P. J. (1970). Gamma function and related functions. In: Handbook of Mathematical Functions with Formulas, Graphs, and Mathematical Tables. Abramowitzn M. and Stegun I.A. (Eds). New York: John Wiley \& Sons, 253-293.

[14] Domma, F., Perri, P. F. (2009). Some developments on the log-Dagum distribution. Statistical Methods and Applications, 18, DOI:10.1007/s10260-007-0091-3, forthcoming.

[15] Embrechts, P., Lindskog, F. and McNeil, A. (2003). Modelling dependence with copulas and applications to risk management. In: Handook of Heavy Tailed Distributions in Finance, Rachev S. T. (Edt). Amsterdam: Elsevier, North Holland.

[16] Engle, R. F. (1982). Autoregressive conditional heteroskedasticity with estimates of the variance of U.K. inflation. Econometrica, 50, 987-1008. 
[17] Fama, E. F. (1971). Efficient capital markets: a review of theory and empirical work. Journal of Finance, 25, 383-417.

[18] Fama, E. F. (1991). Efficient capital markets II. Journal of Finance, 46, 1575-1613.

[19] Fischer, M., Horn, A., Klein, I. (2007). Tukey-type distributions in the context of financial data. Communications in Statistics - Theory and Methods, 36, 23-35.

[20] Fisher, N. I., Switzer, P. (1985). Chi-plots for assessing dependence. Biometrika, 72, 253-265.

[21] Fisher, N.I., Switzer, P. (2001). Graphical assessment of dependence: is a picture worth 100 tests. The American Statisticians, 55, 233-239.

[22] Frees, E. W., Valdez, E. A. (1998). Understanding relationships using copulas. North American Actuarial Journal, 2, 1-25.

[23] Genest, C., Boies, J-C., (2003). Detecting dependence with Kendall plots. The American Statistician, 57, 1-10.

[24] Joe, H. (1997). Multivariate Models and Dependence Concepts, London: Chapman and Hall.

[25] Jones, M. C., Faddy, M. J. (2003). A skew extension of the $t$-distribution, with applications. Journal of the Royal Statistical Society B, 65, 159-174.

[26] Kleiber, C., Kotz, S. (2003). Statistical Size Distribution in Economics and Actuarial Sciences. Hoboken (NJ): John Wiley \& Sons.

[27] Klugman, S. A., Parsa, R. (1999). Fitting bivariate loss distributions with copulas. Insurance: Mathematics and Economics, 24, 139-148.

[28] Hürlimann, W. (2004). Fitting bivariate cumulative returns with copulas. Computational Statistics and Data Analysis, 45, 355-372.

[29] Lehmann, E. L. (1966). Some concepts of dependence. Annals of Mathematical Statistics, 37, 1137-1157.

[30] McDonald, J. B., Xu, Y. J. (1995). A generalization of the beta distribution with applications. Journal of Econometrics, 66, 132-152.

[31] Mendes, B. V. M., Souza, R. M. (2004). Measuring financial risks with copulas. International Review of Financial Analysis, 13, 27-45.

[32] Nelsen, R. B. (1999). An Introduction to Copulas. Lecture Notes in Statistics. New York: Springer.

[33] Pagan, A. (1996). The econometrics of financial markets. Journal of Empirical Finance, 3, 15102.

[34] Rachev, S., Mittnik, S. (2000). Stable paretian models in finance. New York: John Wiley \& Sons.

[35] Savu, C., Ng, W. L. (2005). The SCoD model: analyzing durations with a semiparametric copula approach. International Review of Finance, 5, 55-77. 
[36] Theodossiou, P. (1998). Financial data and the skewed generalized t distribution. Management Science, 44, 1650-1661.

[37] Töyli, J., Faski, K., Kanto, A. (2002). On the shape of asset returns distribution. Communications in Statistics - Simulation and Computations, 31, 489-521. 